\title{
Variability and dust filtration in the transition disk J160421.7-213028 observed in optical scattered light ${ }^{\star}, \star \star$
}

\author{
P. Pinilla ${ }^{1}$, J. de Boer ${ }^{1}$, M. Benisty ${ }^{2,3}$, A. Juhász ${ }^{4}$, M. de Juan Ovelar ${ }^{5}$, C. Dominik ${ }^{6}$, H. Avenhaus ${ }^{7}$, T. Birnstiel ${ }^{8}$, \\ J. H. Girard ${ }^{9}$, N. Huelamo ${ }^{10}$, A. Isella ${ }^{11}$, and J. Milli ${ }^{9}$ \\ 1 Leiden Observatory, Leiden University, PO Box 9513, 2300 RA Leiden, The Netherlands \\ e-mail: pinilla@strw. leidenuniv.nl \\ 2 Univ. Grenoble Alpes, IPAG, 38000 Grenoble, France \\ 3 CNRS, IPAG, 38000 Grenoble, France \\ ${ }^{4}$ Institute of Astronomy, Madingley Road, Cambridge CB3 OHA, UK \\ 5 Astrophysics Research Institute, Liverpool John Moores University,146 Brownlow Hill, Liverpool L3 5RF, UK \\ 6 Astronomical Institute Anton Pannekoek, University of Amsterdam, PO Box 94249, 1090 GE Amsterdam, The Netherlands \\ 7 Departamento de Astronomía, Universidad de Chile, Casilla 36-D, Santiago, Chile \\ 8 Harvard-Smithsonian Center for Astrophysics, 60 Garden Street, Cambridge, MA 02138, USA \\ 9 European Southern Observatory (ESO), Alonso de Córdova 3107, Vitacura, Casilla 19001, Santiago, Chile \\ 10 Centro de Astrobiología (INTA-CSIC), ESAC Campus, PO Box 78, 28691 Villanueva de la Canada, Spain \\ 11 Department of Physics \& Astronomy, Rice University, 6100 Main Street, Houston, TX 77005, USA \\ Received 16 July 2015 / Accepted 22 October 2015
}

\section{ABSTRACT}

\begin{abstract}
Context. Protoplanetary disks around young stars are the birth-sites of planets. Spectral energy distributions and direct images of a subset of disks known as transition disks reveal dust-depleted inner cavities. Some of these disks show asymmetric structures in thermal submillimetre emission and optical scattered light. These structures can be the result of planet(s) or companions embedded in the disk.

Aims. We aim to detect and analyse the scattered light of the transition disk J160421.7-213028, identify disk structures, and compare the results with previous observations of this disk at other wavelengths.

Methods. We obtained and analysed new polarised intensity observations of the transition disk J160421.7-213028 with VLT/SPHERE using the visible light instrument ZIMPOL at $R^{\prime}$-band $(0.626 \mu \mathrm{m})$. We probed the disk gap down to a radius of confidence of $0.1^{\prime \prime}$ $(\sim 15 \mathrm{AU}$ at $145 \mathrm{pc})$. We interpret the results in the context of dust evolution when planets interact with the parental disk.

Results. We observe a gap from 0.1 to $0.3^{\prime \prime}(\sim 15$ to $40 \mathrm{AU})$ and a bright annulus as previously detected by HiCIAO $H$-band observations at $1.65 \mu \mathrm{m}$. The radial width of the annulus is around $40 \mathrm{AU}$, and its centre is at $\sim 61 \mathrm{AU}$ from the central star. The peak of the reflected light at $0.626 \mu \mathrm{m}$ is located $20 \mathrm{AU}$ inward of the cavity detected in the submillimetre. In addition, we detect a dip at a position angle of $\sim 46.2 \pm 5.4^{\circ}$. A dip was also detected with HiCIAO, but located at $\sim 85^{\circ}$. If the dip observed with HiCIAO is the same, this suggests an average dip rotation of $\sim 12^{\circ} /$ year, which is inconsistent with the local Keplerian angular velocity of $\sim 0.8^{\circ} / \mathrm{yr}$ at $\sim 61 \mathrm{AU}$.

Conclusions. The spatial discrepancy in the radial emission in J160421.7-213028 at different wavelengths is consistent with dust filtration at the outer edge of a gap carved by a massive planet. The dip rotation can be interpreted as fast variability of the inner disk and/or the presence of a warp or circumplanetary material of a planet at $\sim 9.6 \mathrm{AU}$.
\end{abstract}

Key words. techniques: high angular resolution - protoplanetary disks

\section{Introduction}

Recent observations of transition disks (TDs) have provided insight into the processes of planet formation and circumstellar disk dissipation (e.g. Espaillat et al. 2014). High-contrast imaging in the optical and near-infrared regime and observations at millimetre wavelengths not only revealed large clear inner cavities, but also several types of structures such as spiral arms, asymmetries, dips, and disk eccentricities (e.g. Garufi et al. 2013; van der Marel et al. 2013; Quanz et al. 2013; Avenhaus et al. 2014; Thalmann et al. 2014; Benisty et al. 2015). Different processes that are not mutually exclusive, can rule the disk evolution and create the observed structures, such as photoevaporation or magneto-rotational instabilities (e.g. Alexander et al. 2014; Flock et al. 2015).

\footnotetext{
* Based on observations performed with VLT/SPHERE under program ID 095.C-0693(A).

$\star \star$ Appendices are available in electronic form at

http://www. aanda.org
}

Spatially resolved observations at different wavelengths are required to distinguish the physical fingerprints that each of these mechanisms leaves on the dust and gas distribution of protoplanetary disks. For example, the spatial segregation between small and large particles, as observed for several TDs (e.g. Garufi et al. 2013), is a natural consequence of filtration effects caused by particle traps (e.g. Rice et al. 2006; Zhu et al. 2012; de Juan Ovelar et al. 2013). One way to form a particle trap is planet-disk interaction: at the outer edge of a planetary gap, a region with positive pressure gradient can stop the fast inward migration of large dust particles (e.g. Pinilla et al. 2012, 2015).

In this Letter, we present polarimetric differential imaging (PDI) of the transition disk around J160421.7-213028 (hereafter J1604), obtained with the subsystem ZIMPOL of the SPHERE instrument of the Very Large Telescope (VLT), at $R^{\prime}$ band $(0.626 \mu \mathrm{m})$. This disk is a member of the Upper Scorpius association (Preibisch \& Zinnecker 1999), which is 5-10 Myr old (Pecaut et al. 2012), and it is located at 

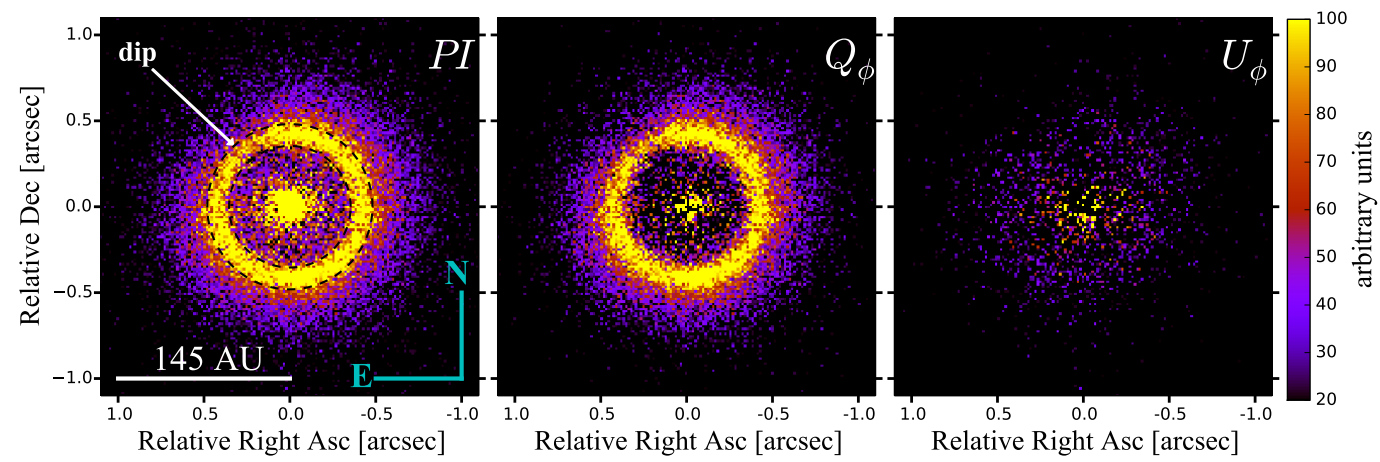

Fig. 1. $R^{\prime}$ band $(0.626 \mu \mathrm{m})$ VLT/SPHERE/ZIMPOL images of J1604 (they are not scaled by $r^{2}$ ). From left to right: polarised intensity (PI), polarcoordinate Stokes parameters $Q_{\phi}$ and $U_{\phi}$ respectively, such that PI $=\sqrt{Q_{\phi}^{2}+U_{\phi}^{2}}$. The clean $U_{\phi}$ image shows that we had an optimal correction for the instrumental polarisation. The colour scale is the same for the three panels; it is linear and in arbitrary units. The dashed lines in the left panel correspond to 0.35 and 0.48 arcsec, which is the region where the azimuthal profile is calculated in Fig. 3 to distinguish the dip.

$\sim 145$ pc (de Zeeuw et al. 1999). The disk is an excellent candidate to identify structures because it has one of the largest cavities reported in TDs and is seen almost face-on $\left(\sim 6^{\circ}\right.$, Mathews et al. 2012). Its cavity was resolved with observations from the Submillimeter Array (SMA; Mathews et al. 2012) and was recently observed with the Atacama Large Millimeter/submillimeter Array (ALMA) in Cycle 0 (Zhang et al. 2014), with a beam size of $0.73^{\prime \prime} \times 0.46^{\prime \prime}(106 \times 67 \mathrm{AU}$ at $145 \mathrm{pc})$. The observations with ALMA showed that the gas cavity is much smaller than the mm-dust cavity (radius of $31 \mathrm{AU}$ inferred from $\mathrm{CO}$ emission vs. $79 \mathrm{AU}$ from the continuum, Zhang et al. 2014). In addition, near-infrared polarised intensity images obtained with HiCIAO at $1.6 \mu \mathrm{m}$ (Mayama et al. 2012) revealed an asymmetric ring of $\sim 63 \mathrm{AU}$ radius, with a dip located at a position angle (PA, measured from north to east) of $85^{\circ}$. A tentative second dip was suggested at PA of $255^{\circ}$.

This Letter is organised as follows. In Sect. 2 we describe the observations and data reduction. The main results from the data analysis and the comparison with previous observations of this disk is presented in Sect. 3. We conclude with the discussion and perspectives in Sect. 4.

\section{Observations and data reduction}

VLT/SPHERE/ZIMPOL observations of J1604 were performed on June 10, 2015, as part of the observing run 095.C-0693(A). We have used field tracking, polarimetric (P2) mode with the $R^{\prime}$ filter $\left(\lambda_{0}=0.626 \mu \mathrm{m}, F W H M=0.148 \mu \mathrm{m}\right)$ for both cameras. Although there is currently no alternative to ZIMPOL for polarimetric imaging of southern targets in the visible, the $R=11.8$ magnitude of J1604 (Cutri et al. 2003) poses a serious challenge for SAXO, the SPHERE extreme adaptive optics "XAO" (Beuzit et al. 2006; Fusco et al. 2014). A beamsplitter divides the visible light of the star between ZIMPOL and the wave front sensor (WFS) of SAXO. Observing in $R^{\prime}$ band allowed us to use the dichroic beamsplitter, which sends all visible light except for the $R$ band to the WFS, thus ensuring an optimal AO correction. During the observations, the seeing conditions were moderate to poor $\left(0.9^{\prime \prime}-1.2^{\prime \prime}\right)$, which caused the Strehl ratio to vary by more than a factor of two. The median Strehl ratio obtained was $\sim 3.5 \%$, resulting in a FWHM of $\sim 53 \times 47$ mas. The observing block was divided into six cycles of the halfwave plate (HWP), during which the HWP moved to four angles $\left(\theta_{\text {hwp }}=0^{\circ} ; 45^{\circ} ; 22.5^{\circ}\right.$; and $\left.67.5^{\circ}\right)$ to measure the two linear Stokes components. For each HWP position, two exposures were taken of $120 \mathrm{~s}$ each, which adds up to 96 minutes of total observing time.
The data reduction is described in detail by De Boer et al., in prep., based on the description of ZIMPOL by Schmid et al. (2012). The pixels of the two detectors have a plate scale of $3.5885 \pm 0.0025$ mas per pixel (Ginski et al., in prep.). We binned the pixels to a size of 14.354 mas. We then substracted the two different states of the ferro-electric liquid crystal (FLC), the 0 and $\pi$ frames (Schmid et al. 2012), the ordinary and extraordinary beams of the polarising beam splitter; and the two matching HWP angles to obtain Stokes $Q$ (for $\theta_{\mathrm{hwp}}=0^{\circ}$ and $45^{\circ}$ ) and $U$ (for $\theta_{\mathrm{hwp}}=22.5^{\circ}$ and $67.5^{\circ}$ ).

Figure 1 shows the polarised intensity PI image and the polar-coordinate Stokes parameters $Q_{\phi}$ and $U_{\phi}$ (Schmid et al. 2006), computed according to

$$
\begin{aligned}
\mathrm{PI} & =\sqrt{Q^{2}+U^{2}}, \\
Q_{\phi} & =Q \times \cos 2 \phi+U \times \sin 2 \phi, \\
U_{\phi} & =Q \times \sin 2 \phi-U \times \cos 2 \phi,
\end{aligned}
$$

where $\phi$ is the position angle.

By measuring the signal over an unpolarised region surrounding the star in the $Q$ and $U$ images, we determined the instrumental polarisation (IP), for which we corrected using the method described by Canovas et al. (2011).

The models of Canovas et al. (2015) show that it is possible for an astrophysical signal to appear in the $U_{\phi}$ images, even when single-scattering dominates. However, this $U_{\phi}$ component only occurs for disks at high inclination $\left(i>40^{\circ}\right)$. Since the disk of $\mathrm{J} 1604$ has an inclination of $i=6 \pm 1.5^{\circ}$ (Mathews et al. 2012), we can use the assumption that the polarised scattered light is entirely tangential and therefore only appear in $Q_{\phi}$, while $U_{\phi}$ should not contain any scattered light signal from the disk. We optimised our IP correction by minimising the $U_{\phi}$ signal and found an optimum when we used an annulus of $10 \leq r \leq 15$ binned pixels.

\section{Results}

\subsection{Radial profile}

Figure 2 shows the overlay of the $R^{\prime}$ band $Q_{\phi}$ reflected light and $880 \mu \mathrm{m}$ continuum map from ALMA Cycle 0 observations (retrieved in the ALMA archive, Zhang et al. 2014). The radial profile of the polarised surface brightness is also illustrated. This profile was obtained by calculating the mean value at each radius from the centre of the $Q_{\phi}$ image, and the error bars correspond to the standard deviation at each position. As a result of poor seeing and moderate AO performance, speckle noise inside a region of $0.1^{\prime \prime}$ surrounding the star still dominates. Therefore, 

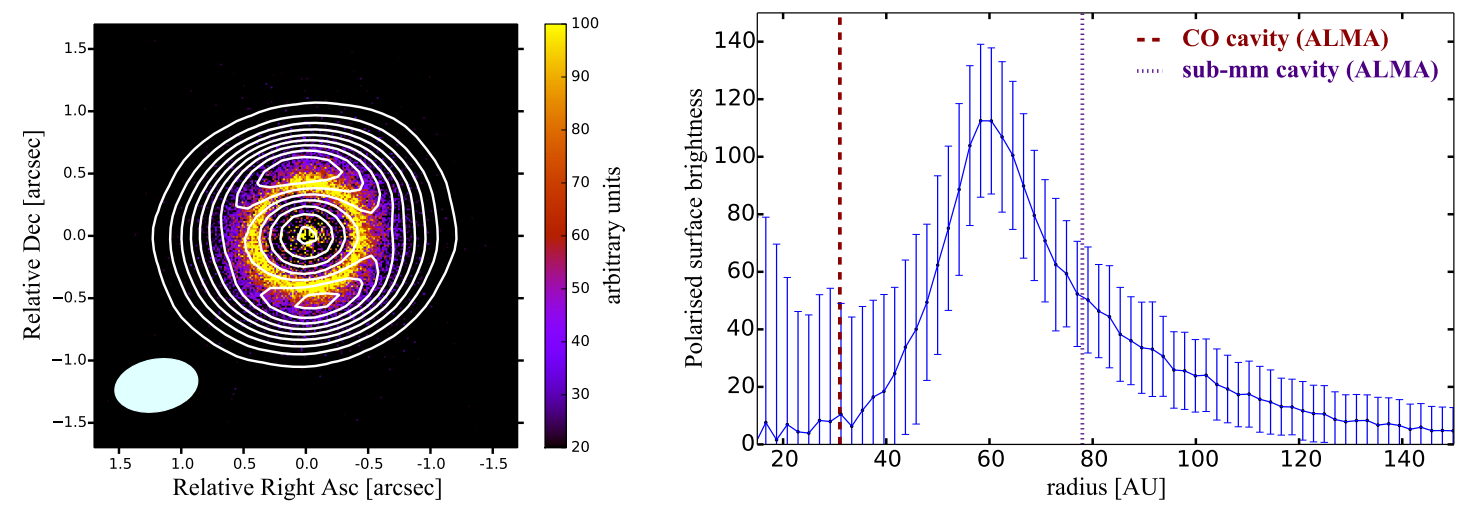

Fig. 2. Left panel: overlay of the $R^{\prime}$ band $(0.626 \mu \mathrm{m}) Q_{\phi}$ reflected light (which is not scaled by $r^{2}$ ) and $880 \mu \mathrm{m}$ map from ALMA Cycle 0 observations (contour lines every 10...90\% peak of the $880 \mu \mathrm{m}$ continuum emission) of J1604. Right panel: radial profile of the polarised surface brightness (arbitrary units), and the comparison with the size of the mm-cavity observed with ALMA at $880 \mu \mathrm{m}$ (Zhang et al. 2014). The cavity radius inferred from $\mathrm{CO} J=3-2$ emission is also displayed. The error bars correspond to the standard deviation at each position from calculating the mean value at each radius from the centre of the image.

we only show the profile from the radius of confidence $\left(>0.1^{\prime \prime}\right.$ corresponding to $>15 \mathrm{AU}$ at $145 \mathrm{pc}$ ).

The radial profile shows that the reflected light at $0.626 \mu \mathrm{m}$ has a gap from 15 to $40 \mathrm{AU}$, and it has a bright annulus from 40 to $80 \mathrm{AU}$. The reflected light extends until $\sim 120 \mathrm{AU}$. We fit a Gaussian profile to the ring emission $\left(a \exp \left[-(x-b)^{2} / 2 c^{2}\right]+d\right)$, from $\sim 40 \mathrm{AU}$ to $\sim 80 \mathrm{AU}$. The centre of the Gaussian $(b)$ and its width $(c)$ were obtained by $\chi^{2}$ minimisation, and the values are $\sim 61.5 \pm 0.3 \mathrm{AU}$ and $\sim 8.5 \pm 0.4 \mathrm{AU}$, respectively. These findings agree with the $H$ band scattered light observations obtained with HiCIAO (Mayama et al. 2012). A comparison between the HiCIAO and ZIMPOL data is shown in Appendix A. Compared with the ALMA observations of the $880 \mu \mathrm{m}$ continuum and CO $J=3-2$ emission, the annulus at $0.626 \mu \mathrm{m}$ lies inside the mm-cavity which has a radius of $\sim 79 \mathrm{AU}$ (Zhang et al. 2014, Fig. 2). The gas cavity radius was inferred around $31 \mathrm{AU}$, but remains unresolved, which is $\sim 9$ AU closer in than the location of the inner radius of the $0.626 \mu$ m annulus.

The surface brightness emission beyond the peak decreases as $\propto r^{-2.92 \pm 0.03}$, indicating a flat and not a flared disk (a more shallow profile is expected for a flared disk, e.g., Whitney \& Hartmann 1992; D'Alessio et al. 1998). However, this profile is more shallow than the surface brightness profile beyond the peak from the HiCIAO data $\left(\propto r^{-4.70 \pm 0.06}\right.$, Fig. A.1).

\subsection{Asymmetric structures}

Figure 3 shows the radial mapping from $0.2-0.6^{\prime \prime}$ of the PI image, which reveals one dip throughout the annulus. Since the disk is almost face-on, the map was not corrected for the inclination, because the projection would make very little difference $(\$ 0.5 \%)$. An azimuthal profile of the polarised surface brightness was obtained by taking the mean values between $0.35-0.48^{\prime \prime}$ after azimuthally binning the data by two degrees, and considering the standard deviation of the data for the error bars. The dip is clearly seen in this azimuthal profile. By fitting a Gaussian profile to the azimuthal profile (i.e. $\left.a \exp \left[-(x-b)^{2} / 2 c^{2}\right]-d\right)$, the best-fit parameter (by $\chi^{2}$ minimisation) found for the location of the dip minimum $(b)$ is $\sim 46.2 \pm 5.4^{\circ}$. Comparing the reflected light at the minimum of the dip and outside the dip, the reflected light is depleted by a factor of $\delta_{\text {dip }} \sim 0.72$. There are no other significant azimuthal changes of the ring morphology for different PA (Appendix B). Mayama et al. (2012) also detected a dip, but at $\sim 85^{\circ}$ and with a higher contrast than our observations $\delta_{\text {dip }} \sim 0.5$. We found no indication of a second dip, which was marginally detected by Mayama et al. (2012) at a PA of $255^{\circ}$. This non-detection might be due to the lower signal-to-noise of our observations.

The HiCIAO and the current data were taken a little more than three years apart (April 11, 2012 and June 10, 2015). Assuming that the dip detected with our observations is the same as was reported by Mayama et al. (2012) at $85^{\circ}$, this would imply that the dip has a fast average rotation speed of around $12.3 \pm 1.7^{\circ} /$ year from east to north (clockwise).

\section{Discussion}

The location of the edge of the gas cavity at 31 AU inferred from observations of CO $J=3-2$ of J1604 with ALMA lies inside the gap seen in scattered light (Fig. 2). In the context of planet disk interaction, when a massive planet opens a gap in the disk, a spatial segregation is expected between the location of the outer edge of the gap in gas and in dust, which is predicted to become larger at longer wavelengths (e.g. Pinilla et al. 2012). This is because the position of the pressure maximum at the outer edge of a gap (i.e. the location where the large particles do not experience radial drift) can be much farther out than the planet position and thus the location of the outer edge of the gap in gas. The fact that in J1604 the edge of the gas cavity lies much closer than the inner edge of the annulus detected in our observations at $R^{\prime}$ band points to a very massive planet or companion.

de Juan Ovelar et al. (2013) predicted the radial profile of emission at different wavelengths after performing radiative transfer together with hydrodynamical and dust-evolution modelling and combined with instrument simulations (including ZIMPOL and ALMA). A large radial segregation between the inner edge of the annulus or "wall" observed with ZIMPOL polarimetric images (defined as the radial location where the flux has increased by half from the minimum in the gap and the peak of the annulus) and the peak of emission at submillimetre $(850 \mu \mathrm{m})$ was predicted for different planet masses and locations (Fig. 8 in de Juan Ovelar et al. 2013). For J1604, the location of the wall of the annulus in $R^{\prime}$ band is $\sim 52 \mathrm{AU}$, implying $r_{\text {wall-ZIMPOL }} / r_{\text {peak-ALMA }} \sim 0.65$, which suggests a massive companion of 5-10 $M_{\text {Jup }}$ mass embedded around 20-40 AU distance from the star. An upper limit of $\sim 18-21 M_{\text {Jup }}$ for a companion at 20-40 AU in J1604 has been inferred from non-redundant aperture mask interferometry (Kraus et al. 2008), which does not contradict our findings. Interestingly, the location of the gas cavity inferred by observations of CO lies also in the same range. 

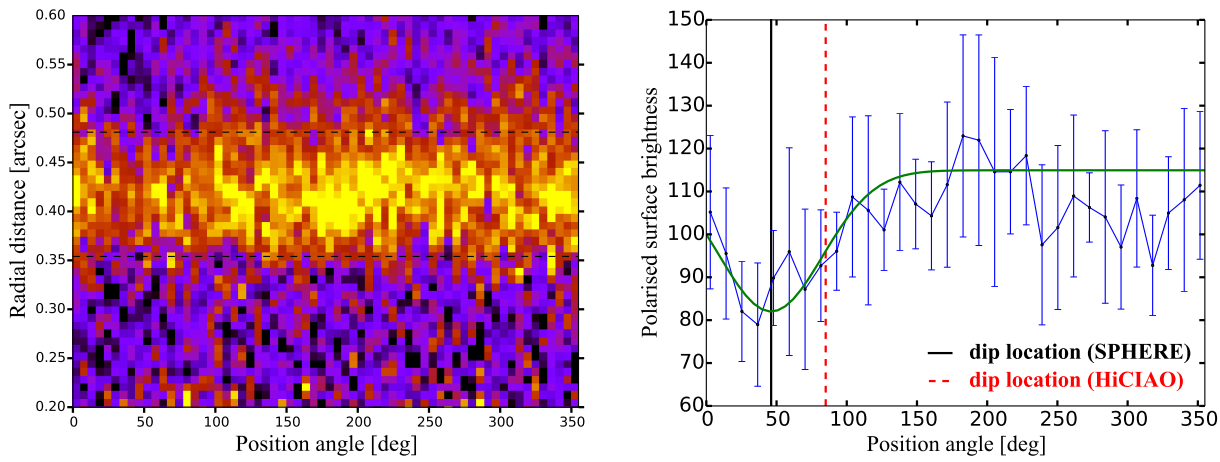

Fig. 3. Left panel: radial mapping from $0.2-0.6$ arcsec of the PI image of J1604 at $R^{\prime}$ band. The colour scale is linear and arbitrary. Right panel: azimuthal profile calculated from the mean values obtained between [0.35-0.48] arcsec (dashed lines in the left panel and in the left panel of Fig. 1). The error bars are from the standard deviation of the data. The solid line represents the best fit of a Gaussian by $\chi^{2}$ minimisation. The vertical lines show the location of the minimum of the dip from our observations and from HiCIAO observations (Mayama et al. 2012).

There is a slight difference of the ring centre between HiCIAO and SPHERE data, which is within the uncertainties of our data (approximately $\sim 1.5$ pixel size, i.e. a shift of $\sim 3$ AU, Fig. A.1). The peak of emission at $R^{\prime}$ and $\mathrm{H}$ band can change for different dust density distributions in the case of planet-disk interaction (shift of $\lesssim 5 \mathrm{AU}$ for very massive planet $\gtrsim 15 M_{\text {Jup }}$, de Juan Ovelar et al. 2013).

If the dip in the ring of J1604 observed with HiCIAO in 2012 is the same structure that we observe, then the dip must be rotating quickly, with an angular speed of $\sim 12^{\circ} /$ year (clockwise as the disk rotation derived from the $\mathrm{CO}$ emission Zhang et al. 2014). The local Keplerian speed at the position of the dip (61 AU) is approximately $\sim 0.8^{\circ} /$ year, much lower than derived from the two observations. Instead, the dip could be the shadow of a structure orbiting much closer to the star. The derived angular velocity corresponds to a Keplerian circular orbit at a distance of only $\sim 9.6 \mathrm{AU}$ from the star (for a $1 M_{\odot}$ star). The nature of the structure creating this shadow is unclear at the moment, it could be a warp in the inner disk regions or a more localised feature such as circumplanetary material of a planet at that location. If the secondary dip in the HiCIAO observations is confirmed, an inclined inner disk might be responsible (Marino et al. 2015). Our observations provide no direct constraints at $10 \mathrm{AU}$ from the star.

If the ring-like structure is created by a planet carving a gap, this planet would be much closer to the ring itself (at 20-40 AU), orbiting more slowly than the dip. The gap-carving planet is therefore unlikely to be associated with the shadowing structure.

It is of course also possible that the dips seen in 2012 and in 2015 are unrelated temporary features, or that the dip rotates in the other direction, that it has rotated over more than 360 degrees, in which cases the derived angular velocity is meaningless and the fact that we see different dips might be related to the observed variability of J1604. While no mid-infrared (MIR) excess was detected in IRS spectra taken with Spitzer (Dahm \& Carpenter 2009), photometric data between 3 and $16 \mu \mathrm{m}$ obtained with the Wide-field Infrared Survey Explorer (WISE) do show MIR excess, suggesting an optically thick narrow ring located close to the dust sublimation radius (Luhman \& Mamajek 2012). The discrepancy between WISE and IRS points to variability of the inner disk. Rapid infrared variability has also been detected in several other disks (e.g. Sitko et al. 2012; Flaherty et al. 2013).

Future high-contrast observations (in a year or more from now) can confirm whether the observed dip is the same in
HiCIAO and our observations and if it rotates with a constant speed; or if the two observed dips are independent events, which would suggest fast inner disk variability.

Acknowledgements. We are grateful to C. P. Dullemond, S. Andrews, and A. Kraus for their feedback and to S. Mayama for sharing the HiCIAO data We thank the VLT team for their help during the observations. P.P. is supported by Koninklijke Nederlandse Akademie van Wetenschappen (KNAW) professor prize to Ewine van Dishoeck. M.B. acknowledges financial support from Programme National de Physique Stellaire (PNPS) of CNRS/INSU, France. A.J. acknowledges the support of the DISCSIM project, grant agreement 341137 funded by the European Research Council under ERC-2013-ADG. H.A. acknowledges financial support from FONDECYT grant 3150643. T.B. acknowledges support from NASA Origins of Solar Systems grant NNX12AJ04G.

\section{References}

Alexander, R., Pascucci, I., Andrews, S., Armitage, P., \& Cieza, L. 2014, Protostars and Planets VI, 475

Avenhaus, H., Quanz, S. P., Meyer, M. R., et al. 2014, ApJ, 790, 56

Benisty, M., Juhasz, A., Boccaletti, A., et al. 2015, A\&A, 578, L6

Beuzit, J.-L., Feldt, M., Dohlen, K., et al. 2006, The Messenger, 125, 29

Canovas, H., Rodenhuis, M., Jeffers, S. V., et al. 2011, A\&A, 531, A102

Canovas, H., Ménard, F., de Boer, J., et al. 2015, A\&A, 582, L7

Cutri, R. M., Skrutskie, M. F., van Dyk, S., et al. 2003, VizieR Online Data Catalog: II/246

Dahm, S. E., \& Carpenter, J. M. 2009, AJ, 137, 4024

D’Alessio, P., Cantö, J., Calvet, N., \& Lizano, S. 1998, ApJ, 500, 411

de Juan Ovelar, M., Min, M., Dominik, C., et al. 2013, A\&A, 560, A111

de Zeeuw, P. T., Hoogerwerf, R. Hubers, J. H. J., et al. 1999, AJ, 117, 354

Espaillat, C., Muzerolle, J., Najita, J., et al. 2014, Protostars and Planets VI, 497

Flaherty, K. M., Muzerolle, J., Rieke, G., et al. 2013, AJ, 145, 66

Flock, M., Ruge, J. P., Dzyurkevich, N., et al. 2015, A\&A, 574, A68

Fusco, T., Sauvage, J.-F., Petit, C., et al. 2014, Proc. SPIE, 9148, 91481

Garufi, A., Quanz, S. P., Avenhaus, H., et al. 2013, A\&A, 560, A105

Kraus, A. L., Ireland, M. J., Martinache, F., \& Lloyd, J. P. 2008, ApJ, 679, 762

Luhman, K. L., \& Mamajek, E. E. 2012, ApJ, 758, 31

Marino, S., Perez, S., \& Casassus, S. 2015, ApJ, 798, L44

Mathews, G. S., Williams, J. P., \& Ménard, F. 2012, ApJ, 753, 59

Mayama, S., Hashimoto, J., Muto, T., et al. 2012, ApJ, 760, L26

Pecaut, M. J., Mamajek, E. E., \& Bubar, E. J. 2012, ApJ, 746, 154

Pinilla, P., Benisty, M., \& Birnstiel, T. 2012, A\&A, 545, A81

Pinilla, P., de Juan Ovelar, M., Ataiee, S., et al. 2015, A\&A, 573, A9

Preibisch, T., \& Zinnecker, H. 1999, AJ, 117, 2381

Quanz, S. P., Avenhaus, H., Buenzli, E., et al. 2013, ApJ, 766, L2

Rice, W. K. M., Armitage, P. J., Wood, K., et al. 2006, MNRAS, 373, 1619

Schmid, H. M., Joos, F., \& Tschan, D. 2006, A\&A, 452, 657

Schmid, H.-M., Downing, M., Roelfsema, R., et al. 2012, Proc. SPIE, 8446 84468

Sitko, M. L. Day, A. N. Kimes, R. L., et al. 2012, ApJ, 745, 29

Thalmann, C., Mulders, G. D., Hodapp, K., et al. 2014, A\&A, 566, A51

van der Marel, N., van Dishoeck, E. F., Bruderer, S., et al. 2013, Science, 340, 1199

Whitney, B. A., \& Hartmann, L. 1992, ApJ, 395, 529

Zhang, K., Isella, A., Carpenter, J. M., \& Blake, G. A. 2014, ApJ, 791, 42

Zhu, Z., Nelson, R. P., Dong, R., et al. 2012, ApJ, 755, 6

Page 5 is available in the electronic edition of the journal at http://www . aanda.org 


\section{Appendix A: Comparison with HiClAO data}

Figure A.1 shows the comparison between the azimuthally averaged radial profile of the polarised surface brightness at $R^{\prime}$ and $H$ band. The data are normalised to the maximum value at $r>0.2^{\prime \prime}$. By fitting a Gaussian profile to the ring emission $\left(a \exp \left[-(x-b)^{2} / 2 c^{2}\right]+d\right)$, the centre of the Gaussian is at $\sim 61.5 \pm 0.3$ and $\sim 64.8 \pm 0.2 \mathrm{AU}$ (for $145 \mathrm{pc}$ distance) for $R^{\prime}$ and $H$ band, respectively. The width of the Gaussian is $\sim 8.5 \pm 0.4$ and $\sim 10.6 \pm 0.9 \mathrm{AU}$ for $R^{\prime}$ and $H$ band respectively. The errors are from the $\chi^{2}$ minimisation and are much smaller than the spatial uncertainty from the observations (1 pixel size $\sim 2$ AU). Fitting a power-law to the brightness profile beyond the location of the peak, the emission drops as $\propto r^{-2.92 \pm 0.03}$ and $\propto r^{-4.70 \pm 0.06}$ for $R^{\prime}$ and $H$ band, respectively.

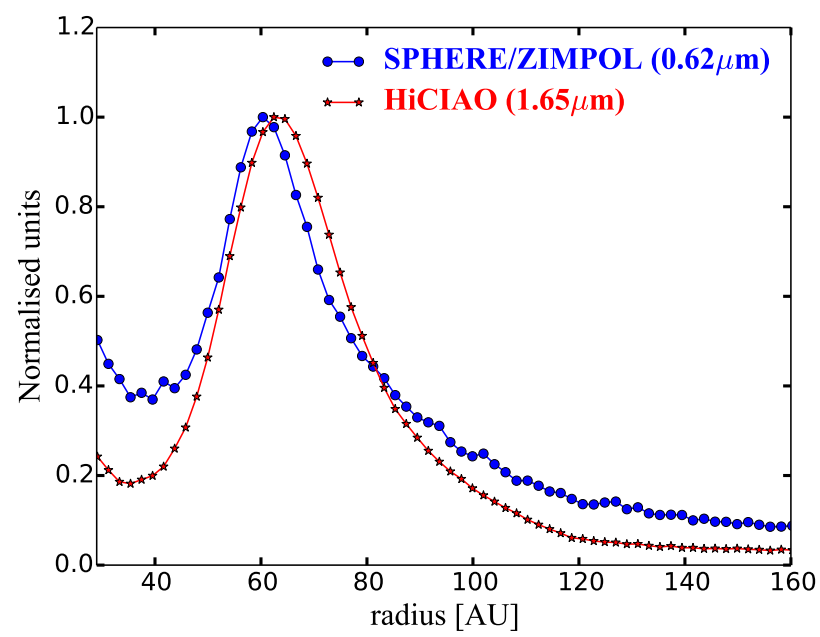

Fig. A.1. Comparison between the azimuthally averaged radial profile of the polarised surface brightness at $R^{\prime}$ and $H$ band. The data are normalised to the maximum value at $r>0.2^{\prime \prime}$. Error bars are omitted for better readability; typical values are $40 \%$ and $30 \%$ of the mean value for $R^{\prime}$ and $H$ band respectively.

\section{Appendix B: Ring shape at different azimuthal cuts}

Figure B.1 shows the radial profile of the azimuthally averaged surface brightness over four bins of PA. A Gaussian profile is fitted to each case; the width and centre of the Gaussians are summarised in Table B.1. The fitting results show that there are no significant azimuthal variations of the ring within the uncertainties of the data (pixel size $\sim 2 \mathrm{AU}$ ).

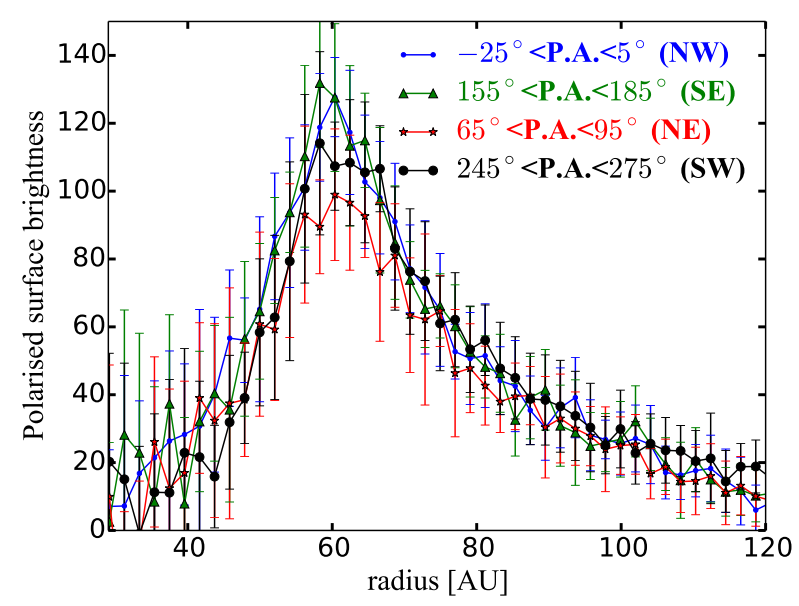

Fig. B.1. Radial profile of the polarised surface brightness obtained assuming four different cuts of the PA.

Table B.1. Centre and width of the Gaussians fits for the profiles in Fig. B.1.

\begin{tabular}{ccc}
\hline \hline Cut & $\begin{array}{c}b \text { (centre) } \\
{[\mathrm{AU}]}\end{array}$ & $\begin{array}{c}c \text { (width) } \\
{[\mathrm{AU}]}\end{array}$ \\
\hline NW & 61.4 & 8.7 \\
SE & 61.4 & 8.4 \\
NE & 62.7 & 9.1 \\
SW & 64.0 & 9.2 \\
\hline
\end{tabular}

Notes. The statistical errors are omitted since they are smaller than the spatial uncertainty from the observations (1 pixel size $\sim 2 \mathrm{AU}$ ). 